\title{
Constraining the evolution of dark energy with type la supernovae and gamma-ray bursts
}

\author{
Shi Qi ${ }^{1,4}$, Fa-Yin Wang ${ }^{2}$, and Tan $\mathrm{Lu}^{3,4}$ \\ 1 Department of Physics, Nanjing University, Nanjing 210093, PR China \\ e-mail: qishi11@gmail.com \\ 2 Department of Astronomy, Nanjing University, Nanjing 210093, PR China \\ e-mail: fayinwang@nju.edu.cn \\ 3 Purple Mountain Observatory, Chinese Academy of Sciences, Nanjing 210008, PR China \\ e-mail: t.lu@pmo.ac.cn \\ 4 Joint Center for Particle, Nuclear Physics and Cosmology, Nanjing University - Purple Mountain Observatory, Nanjing 210093, \\ PR China
}

Received 26 December 2007 / Accepted 19 February 2008

\begin{abstract}
Aims. The behavior of the dark energy equation of state (EOS) is crucial in distinguishing different cosmological models. With a model independent approach, we constrain the possible evolution of the dark energy EOS.

Methods. Gamma-ray bursts (GRBs) of redshifts up to $z>6$ are used, in addition to type Ia supernovae (SNe Ia). We separate the redshifts into 4 bins and assume a constant EOS parameter for dark energy in each bin. The EOS parameters are decorrelated by diagonalizing the covariance matrix. And the evolution of dark energy is estimated out of the uncorrelated EOS parameters.

Results. By including GRB luminosity data, we significantly reduce the confidence interval of the uncorrelated EOS parameter whose contribution mostly comes from the redshift bin of $0.5<z<1.8$. At high redshift where we only have GRBs, the constraints on the dark energy EOS are still very weak. However, we can see an obvious cut at about zero in the probability plot of the EOS parameter, from which we can infer that the ratio of dark energy to matter most probably continues to decrease beyond redshift 1.8 . We carried out analyses with and without including the latest BAO measurements, which themselves favor a dark energy EOS of $w<-1$. If they are included, the results show some evidence of an evolving dark energy EOS. If not included, however, the results are consistent with the cosmological constant within $1 \sigma$ for redshift $0<z \lesssim 0.5$ and $2 \sigma$ for $0.5 \lesssim z<1.8$.
\end{abstract}

Key words. cosmological parameters - supernovae: general - gamma rays: bursts

\section{Introduction}

Unexpected accelerating expansion of the universe was first discovered by observing type Ia supernovae (SNe Ia) (Riess et al. 1998; Perlmutter et al. 1999). This acceleration is attributed to dark energy, whose presence was corroborated later by other independent sources including the WMAP and other observations of the CMB (Spergel et al. 2003), X-ray clusters (Allen et al. 2002), etc. With more observational data available (e.g. Hawkins et al. 2003; Abazajian et al. 2003; Spergel et al. 2007; Riess et al. 2007; Wood-Vasey et al. 2007; Davis et al. 2007; Schaefer 2007), we are getting more stringent constraints on the nature of dark energy; nevertheless, the underlying physics of dark energy remains mysterious. In addition to the cosmological constant, many other dark energy models have been suggested, including models of scalar fields (see Copeland et al. 2006, for a recent review) and modification of general relativity (see for example Deffayet 2001; Binetruy et al. 2000; Maartens 2007; Capozziello et al. 2003; Dvali et al. 2000; Carroll et al. 2004).

Measuring the expansion history directly may be the best way to constrain the properties of dark energy. To measure the expansion history, we need standard candles at different redshifts. SNe Ia, which are now viewed as nearly ideal standard candles, have played an important role in constraining cosmological parameters. We now have 192 samples of SN Ia (Riess et al. 2007; Wood-Vasey et al. 2007; Davis et al. 2007) that can be used to determine the expansion history. And the proposed SNAP satellite ${ }^{1}$ (see for example Aldering et al. 2004) will add about 2000 samples per year. Increasing SN Ia samples will provide more and more precise description of the cosmic expansion. However, the redshift of the present $192 \mathrm{SNe}$ Ia ranges only up to about 1.7 and the mean redshift is about 0.5 . They cannot provide any information on the cosmic expansion beyond redshift 1.7. Here gamma-ray bursts (GRBs) come in and fill the void. With their higher luminosities, GRBs are visible across much greater distances than supernovae. The presently available 69 compiled GRBs (Schaefer 2007) extend the redshift to $z>6$ and the mean redshift is about 2.1. After being calibrated with luminosity relations, GRBs may be used as standard candles to provide information on cosmic expansion at high redshift and, at the same time, to tighten the constraints on cosmic expansion at low redshift. See, for example, Dai et al. (2004), Ghirlanda et al. (2004), Di Girolamo et al. (2005), Firmani et al. (2005), Friedman \& Bloom (2005), Lamb et al. (2005), Liang \& Zhang (2005), Xu et al. (2005), Wang \& Dai (2006),

\footnotetext{
1 See http://www.snap.lbl.gov/
} 
Li et al. (2008), Su et al. (2006), Schaefer (2007), Wright (2007), and Wang et al. (2007) for works on GRB cosmology.

Among parameters that describe the properties of dark energy, the equation of state (EOS) is the most important. Whether and how it evolves with time is crucial in distinguishing different cosmological models. Due to not understanding of the behaviors of dark energy, simple parametric forms such as $w(z)=w_{0}+w^{\prime} z$ (Cooray \& Huterer 1999) and $w(z)=w_{0}+$ $w_{a} z /(1+z)($ Chevallier \& Polarski 2001; Linder 2003) have been proposed for studying the possible evolution of dark energy. However, a simple parameterization itself greatly restricts the allowed wandering of $w(z)$, and is equivalent to a strong prior on the nature of dark energy (Riess et al. 2007). To avoid any strong prior before comparing data, one can utilize an alternative approach in which uncorrelated estimates are made of discrete $w(z)$ of different redshifts. This approach was proposed by Huterer \& Starkman (2003) and Huterer \& Cooray (2005) and has been adopted in previous analyses using SNe Ia (Riess et al. 2007; Sullivan et al. 2007a).

In this work, we apply this approach to GRB luminosity data (Schaefer 2007), in addition to SN Ia data (Riess et al. 2007; Wood-Vasey et al. 2007; Davis et al. 2007), and compare our results with those in the previous work that does not include GRB luminosity data (Sullivan et al. 2007a). We first briefly review the techniques for uncorrelated estimates of dark energy evolution in Sect. 2. The observational data and how they are included in the data analysis are described in Sect. 3. We present our results in Sect. 4, followed by a summary in Sect. 5 .

\section{Methodology}

Standard candles impose constraints on cosmological parameters essentially through a comparison of the luminosity distance from observation with that from theoretical models. Observationally, the luminosity distance is given by

$d_{L}=\left(\frac{L}{4 \pi F}\right)^{1 / 2}$,

where $L$ and $F$ are the luminosity of the standard candles and the observed flux, respectively. Theoretically, the luminosity distance $d_{L}(z)$ depends on the geometry of the universe, i.e. the sign of $\Omega_{k}$, and is given by

$d_{L}(z)=(1+z) \frac{c}{H_{0}} \times \begin{cases}\frac{1}{\sqrt{\Omega_{k}}} \sinh \left(\sqrt{\left|\Omega_{k}\right|} \int_{0}^{z} \frac{\mathrm{d} \tilde{z}}{E(\tilde{z})}\right) & \text { if } \Omega_{k}>0 \\ \int_{0}^{z} \frac{\mathrm{d} \tilde{z}}{E(\tilde{z})} & \text { if } \Omega_{k}=0 \\ \frac{1}{\sqrt{\mid \Omega_{k}}} \sin \left(\sqrt{\left|\Omega_{k}\right|} \int_{0}^{z} \frac{\mathrm{d} \tilde{z}}{E(\tilde{z})}\right) & \text { if } \Omega_{k}<0,\end{cases}$

where

$$
\begin{array}{r}
E(z)=\left[\Omega_{\mathrm{m}}(1+z)^{3}+\Omega_{x} f(z)+\Omega_{k}(1+z)^{2}\right]^{1 / 2}, \\
\Omega_{\mathrm{m}}+\Omega_{x}+\Omega_{k}=1
\end{array}
$$

and

$f(z)=\exp \left[3 \int_{0}^{z} \frac{1+w(\tilde{z})}{1+\tilde{z}} \mathrm{~d} \tilde{z}\right]$.

Dark energy parameterization schemes enter through $f(z)$. For the case where EOS is piecewise constant in redshift, $f(z)$ can be rewritten as (Sullivan et al. 2007a)

$f\left(z_{n-1}<z \leq z_{n}\right)=(1+z)^{3\left(1+w_{n}\right)} \prod_{i=0}^{n-1}\left(1+z_{i}\right)^{3\left(w_{i}-w_{i+1}\right)}$, where $w_{i}$ is the EOS parameter in the $i$ th redshift bin defined by an upper boundary at $z_{i}$, and the zeroth bin is defined as $z_{0}=0$. In order to compare with previous analysis (Sullivan et al. 2007a), we define the first three redshift bins to be the same as those used by Sullivan et al. (2007a) by setting $z_{1}=0.2, z_{2}=0.5$, and $z_{3}=$ 1.8. The fourth bin is defined by $z_{4}=7$ to include GRBs. We carry out our analyses under two different assumptions about the high redshift (redshift greater than $z_{4}=7$ in our case) behavior of dark energy, i.e. the so-called (see Riess et al. 2007) "weak" prior, which makes no assumptions about $w(z)$ at $z>7$ and the "strong" prior, which assumes $w(z)=-1$ at $z>7$.

In this paper we adopt $\chi^{2}$ statistic to estimate parameters. For a physical quantity $\xi$ with experimentally measured value $\xi_{o}$, standard deviation $\sigma_{\xi}$, and theoretically predicted value $\xi_{t}(\theta)$, where $\theta$ is a collection of parameters needed to calculate the theoretical value, the $\chi^{2}$ value is given by

$\chi_{\xi}^{2}(\theta)=\frac{\left(\xi_{t}(\theta)-\xi_{o}\right)^{2}}{\sigma_{\xi}^{2}}$

and the total $\chi^{2}$ is the sum of all $\chi_{\xi}^{2}$ s, i.e.

$\chi^{2}(\theta)=\sum_{\xi} \chi_{\xi}^{2}(\theta)$

The likelihood function is then proportional to $\exp \left(-\chi^{2}(\theta) / 2\right)$, which produces the posterior probability when multiplied by the prior probability of $\theta$. In the case of our analysis, the calculation of $\chi^{2}$ s for different observational data is described in Sect. 3. According to the posterior probability derived in this way, Markov chains are generated through the Monte-Carlo algorithm to study the statistical properties of the parameters. In this paper, we focus on the EOS parameters by marginalizing the others.

As mentioned above, in the process of constraining cosmological parameters, standard candles play this role by providing the luminosity distances at certain redshifts. However, the luminosity distance depends on the integration of the behavior of the dark energy over redshift, so the estimates of the dark energy EOS parameters $w_{i}$ at high redshift depend on those at low redshift. In other words, the EOS parameters $w_{i}$ are correlated in the sense that the covariance matrix,

$\boldsymbol{C}=\left\langle\boldsymbol{w} \boldsymbol{w}^{\mathrm{T}}\right\rangle-\langle\boldsymbol{w}\rangle\left\langle\boldsymbol{w}^{\mathrm{T}}\right\rangle$,

is not diagonal. In the above equation, the $w$ is a vector with components $w_{i}$ and the average is calculated by letting $\boldsymbol{w}$ run over the Markov chain. We can obtain a set of decorrelated parameters $\widetilde{w}_{i}$ through diagonalization of the covariance matrix by choosing an appropriate transformation

$\widetilde{w}=T w$.

There can be different choices for $\boldsymbol{T}$. In this paper we use the transformation advocated by Huterer \& Cooray (2005) (see below). First we define the Fisher matrix

$\boldsymbol{F} \equiv \boldsymbol{C}^{-1}=\boldsymbol{O}^{\mathrm{T}} \boldsymbol{\Lambda} \boldsymbol{O}$,

and then the transformation matrix $\boldsymbol{T}$ is given by

$\boldsymbol{T}=\boldsymbol{O}^{\mathrm{T}} \boldsymbol{\Lambda}^{\frac{1}{2}} \boldsymbol{O}$

except that the rows of the matrix $\boldsymbol{T}$ are normalized such that

$\sum_{j} T_{i j}=1$ 


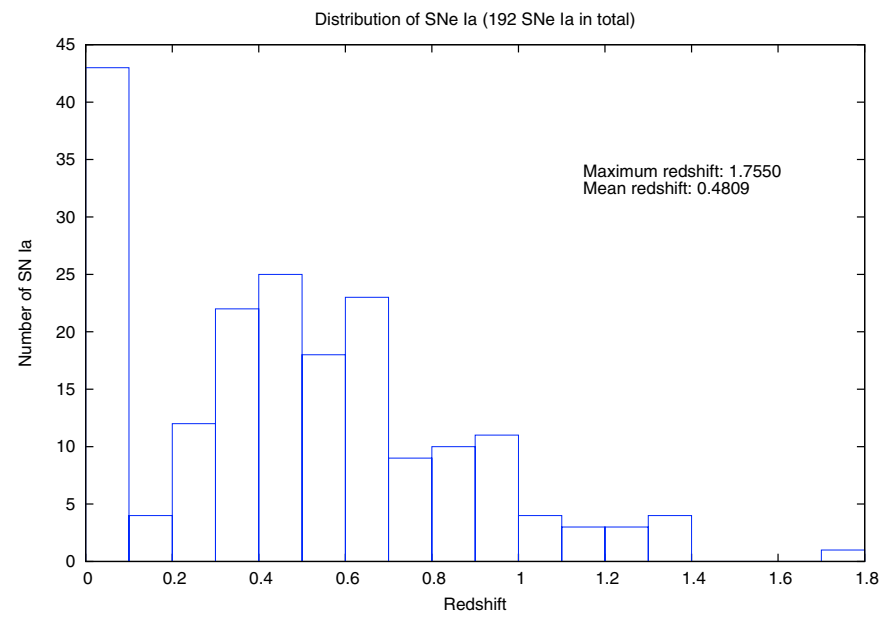

Fig. 1. Distribution of SN Ia samples versus redshift.

The advantage of this transformation is that the weights (rows of $\boldsymbol{T}$ ) are positive almost everywhere and localized in redshift fairly well, so the uncorrelated EOS parameters $\widetilde{w}_{i}$ are easy to interpret intuitively (Huterer \& Cooray 2005).

\section{Observational data}

To constrain the dark energy EOS, we have made use of observational data described below.

\subsection{Type la supernovae}

Recently compiled SN Ia data (Riess et al. 2007; Wood-Vasey et al. 2007; Davis et al. 2007 include 45 nearby supernovae (Hamuy et al. 1996; Riess et al. 1999; Jha et al. 2006), 60 ESSENCE supernovae (Wood-Vasey et al. 2007), 57 SNLS supernovae (Astier et al. 2006), and 30 HST supernovae (Riess et al. 2007). Figure 1 shows the distribution of these SN Ia samples versus redshift. The $\chi^{2}$ value for SNe Ia is

$\chi_{\mathrm{SN}}^{2}=\sum_{i} \frac{\left(\mu_{\mathrm{p}, i}-\mu_{\mathrm{o}, i}\right)^{2}}{\sigma_{i}^{2}+\sigma_{\mathrm{int}}^{2}}$,

where $\mu_{\mathrm{o}, i}$ and $\mu_{\mathrm{p}, i}$ are the observed and theoretically predicted distance modulus of SN Ia, which is defined by $\mu=5 \log d_{\mathrm{L}}+25$ with the luminosity distance $d_{\mathrm{L}}$ in unit of megaparsec and $\sigma_{\text {int }}$ is the intrinsic dispersion.

\subsection{Gamma-ray bursts}

Besides SNe Ia, GRB luminosity data is another main observational constraint we used. As mentioned before, GRBs are complementary to SNe Ia at high redshifts. We include GRBs presented by Schaefer (2007) (see Fig. 2 for the distribution of these GRBs versus redshift) in our analysis by utilizing the five luminosity relations, i.e. the connections between measurable parameters of the light curves and/or spectra and GRB luminosity: $\tau_{\text {lag }}-L, V-L, E_{\text {peak }}-L, E_{\text {peak }}-E_{\gamma}$ and $\tau_{\mathrm{RT}}-L$

$$
\begin{aligned}
& \log \frac{L}{1 \mathrm{erg} \mathrm{s}^{-1}}=a_{1}+b_{1} \log \left[\frac{\tau_{\text {lag }}(1+z)^{-1}}{0.1 \mathrm{~s}}\right], \\
& \log \frac{L}{1 \mathrm{erg} \mathrm{s}^{-1}}=a_{2}+b_{2} \log \left[\frac{V(1+z)}{0.02}\right],
\end{aligned}
$$

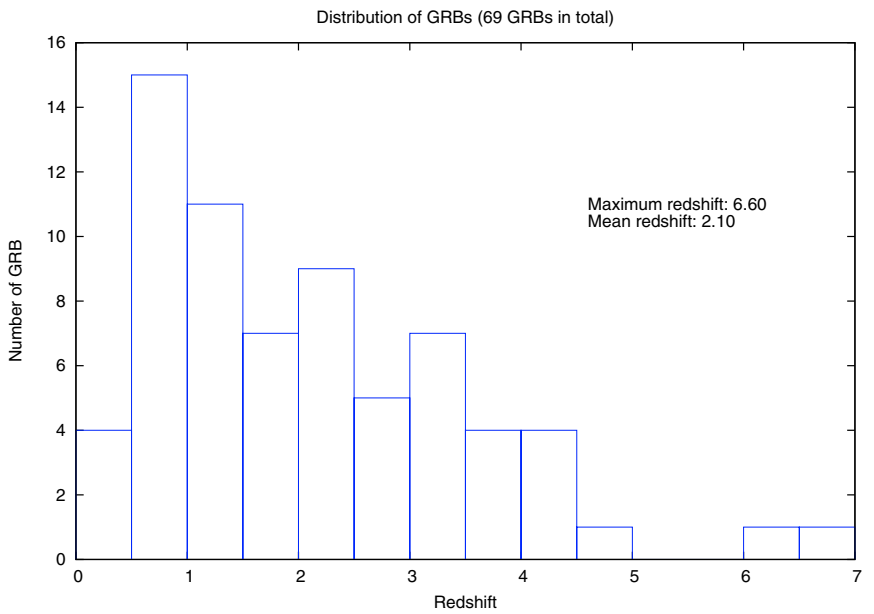

Fig. 2. Distribution of GRB samples versus redshift.

$$
\begin{aligned}
\log \frac{L}{1 \mathrm{erg} \mathrm{s}^{-1}} & =a_{3}+b_{3} \log \left[\frac{E_{\text {peak }}}{300 \mathrm{keV}}\right], \\
\log \frac{E_{\gamma}}{1 \mathrm{erg}} & =a_{4}+b_{4} \log \left[\frac{E_{\text {peak }}}{300 \mathrm{keV}}\right], \\
\log \frac{L}{1 \mathrm{erg} \mathrm{s}^{-1}} & =a_{5}+b_{5} \log \left[\frac{\tau_{\mathrm{RT}}(1+z)^{-1}}{0.1 \mathrm{~s}}\right] .
\end{aligned}
$$

Throughout this paper, by GRB luminosity data we refer to the GRBs' observational data related to such luminosity relations. It is worth mentioning that these relations may be correlated. As discussed in Schaefer (2007), there is one significant correlation between the $V$ - $L$ and $\tau_{\mathrm{RT}}-L$ relations with the correlation coefficient equaling 0.53 . However, even for this correlation, ignoring it only causes a $4 \%$ underestimate in the standard error of the average distance modulus (Schaefer 2007), so in our analysis we safely ignore the correlations and simply add the contributions from each relation (see Eq. (21) below).

There are significant differences between SNe Ia and GRBs on the calibration. For SNe Ia, the calibration is done with nearby events and is therefore independent of cosmological parameters. The luminosity relations obtained in the calibration are applied to high-redshift events to derive the luminosity of SNe Ia, then used to constrain cosmological parameters. In this procedure, the calibration and the constraining of cosmological parameters are done separately. In contrast to $\mathrm{SNe}$ Ia, to constrain cosmological parameters using GRBs, we need to know the luminosity relations of GRBs (Eqs. (14)-(18)), i.e. to know the values of $a_{1}-a_{5}$ and $b_{1}-b_{5}$; consequently, we need the luminosity $L$ and the total collimation-corrected energy $E_{\gamma}$ of GRBs, which are converted respectively from the bolometric peak flux $P_{\text {bolo }}$ and the bolometric fluence $S_{\text {bolo }}$ of GRBs through the relations

$$
\begin{aligned}
L & =4 \pi d_{\mathrm{L}}^{2} P_{\text {bolo }}, \\
E_{\gamma} & =E_{\gamma, \text { iso }} F_{\text {beam }}=4 \pi d_{\mathrm{L}}^{2} S_{\text {bolo }}(1+z)^{-1} F_{\text {beam }} .
\end{aligned}
$$

The conversion depends on cosmological parameters because the luminosity distance $d_{\mathrm{L}}$ depends on cosmological models. As a result, the calibration and the constraining of cosmological parameters are mixed for GRBs; i.e., we need to simultaneously fit calibration parameters of GRBs and cosmological parameters. 
Based on the above discussions, the $\chi^{2}$ value for GRBs is calculated by

$$
\begin{aligned}
\chi_{\mathrm{GRB}}^{2}= & \sum_{i} \frac{\left\{\log \frac{L_{i}}{1 \mathrm{erg} \mathrm{s}^{-1}}-a_{1}-b_{1} \log \left[\frac{\tau_{\text {lag }, i}\left(1+z_{i}\right)^{-1}}{0.1 \mathrm{~s}}\right]\right\}^{2}}{\sigma_{1}^{2}} \\
& +\sum_{i} \frac{\left\{\log \frac{L_{i}}{1 \operatorname{erg~s}^{-1}}-a_{2}-b_{2} \log \left[\frac{V_{i}\left(1+z_{i}\right)}{0.02}\right]\right\}^{2}}{\sigma_{2}^{2}} \\
& +\sum_{i} \frac{\left\{\log \frac{L_{i}}{1 \operatorname{erg~s}^{-1}}-a_{3}-b_{3} \log \left[\frac{E_{\text {peak }, i}}{300 \mathrm{keV}}\right]\right\}^{2}}{\sigma_{3}^{2}} \\
& +\sum_{i} \frac{\left\{\log \frac{E_{\gamma, i}}{1 \mathrm{erg}}-a_{4}-b_{4} \log \left[\frac{E_{\text {peak }, i}}{300 \mathrm{keV}}\right]\right\}^{2}}{\sigma_{4}^{2}} \\
& +\sum_{i} \frac{\left\{\log \frac{L_{i}}{1 \mathrm{erg} \mathrm{s} \mathrm{s}^{-1}}-a_{5}-b_{5} \log \left[\frac{\tau_{\mathrm{RT}, i}\left(1+z_{i}\right)^{-1}}{0.1 \mathrm{~s}}\right]\right\}^{2}}{\sigma_{5}^{2}}
\end{aligned}
$$

where $L_{i}$ and $E_{\gamma, i}$ are derived using Eqs. (19) and (20). The summations run over the GRBs with the corresponding luminosity indicator observed. We use the systematic errors estimated by (Schaefer 2007) that account for the scatter of the log-log plots of the luminosity versus the luminosity indicators as $\sigma_{1}-$ $\sigma_{5}$ in our analysis. Apparently, $\chi_{\mathrm{GRB}}^{2}$ is a function of calibration parameters $a_{1}-a_{5}, b_{1}-b_{5}$ and cosmological parameters that enter through the luminosity distance $d_{\mathrm{L}}$.

\subsection{Other data}

In addition to SNe Ia and GRBs, we have also used the constraints below following previous analyses (Riess et al. 2007; Sullivan et al. 2007a)

- Constraints on dimensionless mass densities: the SDSS large-scale structure measurements give the constraint on local mass density in terms of $\Omega_{\mathrm{m}} h=0.213 \pm 0.023$ (Tegmark et al. 2004). The WMAP three-year data combined with the HST key project constraint on the Hubble constant gives $\Omega_{k}=-0.014 \pm 0.017$ (Spergel et al. 2007).

- The SDSS luminous red galaxy, baryon acoustic oscillation $(B A O)$ distance parameter to $z_{\mathrm{BAO}}=0.35: A \equiv$ $\frac{\sqrt{\Omega_{\mathrm{m}} H_{0}^{2}}}{c z_{\mathrm{BAO}}}\left[r^{2}\left(z_{\mathrm{BAO}}\right) \frac{c z_{\mathrm{BAO}}}{H_{0} E\left(z_{\mathrm{BAO}}\right)}\right]^{1 / 3}$, where $r(z)=d_{\mathrm{L}}(z) /(1+z)$. $A=0.469\left(\frac{n}{0.98}\right)^{-0.35} \pm 0.017$ from Eisenstein et al. (2005) and the three-year WMAP results give $n=0.95$ (Spergel et al. 2007).

- The distance to last scattering, $z=1089$ : if nonzero cosmic curvature is allowed as we do in our analysis, the three-year WMAP data (Spergel et al. 2007) gives the shift parameter $R_{\mathrm{CMB}}=\frac{\sqrt{\Omega_{\mathrm{m}} H_{0}^{2}}}{c} r\left(z_{\mathrm{CMB}}\right)=1.71 \pm 0.03$ (Wang \& Mukherjee 2007).

- The distance ratio between $z_{\mathrm{BAO}}=0.35$ and $z_{\mathrm{CMB}}=1089$ :

$$
R_{0.35}=\frac{\left[r^{2}\left(z_{\mathrm{BAO}}\right) \frac{c z_{\mathrm{BAO}}}{H_{0} E\left(z_{\mathrm{BAO}}\right)}\right]^{1 / 3}}{r\left(z_{\mathrm{CMB}}\right)} .
$$

The SDSS BAO analysis (Eisenstein et al. 2005) gives $R_{0.35}=0.0979 \pm 0.0036$.

The corresponding $\chi^{2}$ for these constraints are directly calculated using Eq. (6).
Table 1. Number of SNe Ia and GRBs that fall into the four bins.

\begin{tabular}{ccccc}
\hline \hline Bin & 1 & 2 & 3 & 4 \\
\hline Redshift range & $0-0.2$ & $0.2-0.5$ & $0.5-1.8$ & $1.8-7$ \\
Number of SNe Ia & 47 & 59 & 86 & 0 \\
Number of GRBs & 1 & 3 & 32 & 33 \\
Total number & 48 & 62 & 118 & 33 \\
\hline
\end{tabular}

We have also studied the dark energy EOS evolution with the above BAO constraints replaced by the latest BAO measurements presented in Percival et al. (2007), for which the $\chi^{2}$ value is (Percival et al. 2007)

$\chi_{\mathrm{BAO}}^{2}=\boldsymbol{X}_{\mathrm{BAO}}^{\mathrm{T}} \boldsymbol{C}_{\mathrm{BAO}}^{-1} \boldsymbol{X}_{\mathrm{BAO}}$

where

$\boldsymbol{X}_{\mathrm{BAO}}=\left(\begin{array}{c}\frac{r_{\mathrm{s}}}{D_{V_{\mathrm{s}}}(0.2)}-0.1980 \\ \frac{D_{V}(0.35)}{V_{\mathrm{s}}}-0.1094\end{array}\right)$

with $r_{\mathrm{s}}$ the comoving sound horizon at recombination and

$\boldsymbol{C}_{\mathrm{BAO}}^{-1}=\left(\begin{array}{cc}35059 & -24031 \\ -24031 & 108300\end{array}\right)$

This constraint itself favors a dark energy EOS of $w<-1$ (Percival et al. 2007).

\section{Results}

Figures 3 and 4 show our results for the weak prior and strong prior respectively. For these two figures, we have included subsets of data from Sect. 3.3 same as that are used in Sullivan et al. (2007a) besides SNe Ia. For the results presented in Fig. 5, the BAO constraints are updated with the latest measurements (Percival et al. 2007), see Eqs. (23)-(25). A comparison between Figs. 3 and 4 shows that the results are insensitive to the priors, i.e. insensitive to whether $w(z>7)=-1$ is assumed or not for dark energy.

Since Figs. 3 and 4 only differ from results derived by Sullivan et al. (2007a) in that we include GRB luminosity data, comparisons of Figs. 3 and 4 with figures in Sullivan et al. (2007a) demonstrate the improvement made by including GRBs. We find that there is little improvement in $\widetilde{w}_{1}$ and $\widetilde{w}_{2}$. This is because at low redshift, where we have both SNe Ia and GRBs, there are fewer GRBs than that of SNe Ia (see Table 1, in the first two bins the number of GRBs is negligible compared with that of SNe Ia); at the same time, the contributions to $\widetilde{w}_{1}$ and $\widetilde{w}_{2}$ from high redshift, where we have a considerable number of GRB samples (see Table 1), are too small (see the weight histograms in Figs. 3 and 4 ) to improve constraints on $\widetilde{w}_{1}$ and $\widetilde{w}_{2}$ significantly. The most significant improvement lies in $\widetilde{w}_{3}$, whose contribution mostly comes from the third bin, where we have several GRBs (see Table 1 ). The $1 \sigma$ confidence interval of $\widetilde{w}_{3}$ with GRBs included is less than one third of that presented in Sullivan et al. (2007a) without including GRB luminosity data.

For Figs. 3 and $4, \widetilde{w}_{1}$ and $\widetilde{w}_{2}$ are consistent with the cosmological constant within $1 \sigma$, and $\widetilde{w}_{3}$ consistent within $2 \sigma$. While in Fig. 5, for which the latest BAO measurements are used instead, the cosmological constant lies outside of the $1 \sigma$ confidence intervals of $\widetilde{w}_{1}$ and $\widetilde{w}_{2}$, and outside the $2 \sigma$ confidence interval of $\widetilde{w}_{3}$, though still inside the $2 \sigma$ confidence intervals of $\widetilde{w}_{1}$ and $\widetilde{w}_{2}$. These results show some evidence of an evolving dark energy EOS. This is not surprising provided that the latest BAO measurements themselves favor a dark energy EOS of 

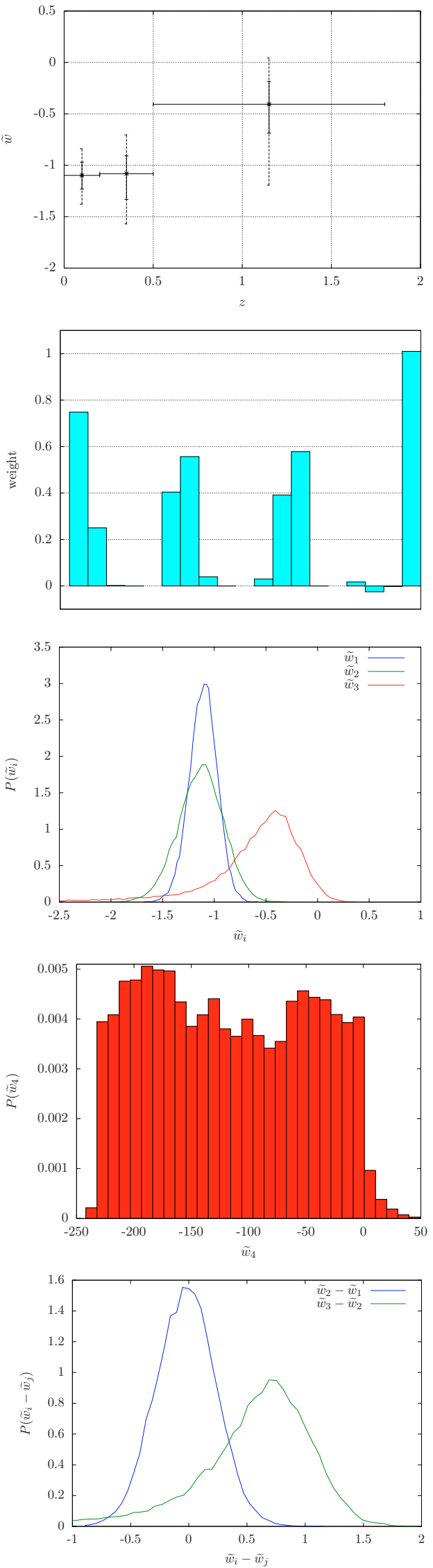

Fig. 3. Estimates of the uncorrelated dark energy EOS parameters using the weak prior. In turn are the plots of $\widetilde{w}_{i}(i=1-3)$ versus redshift, window functions of $\widetilde{w}_{i}(i=1-4)$ with respect to the 4 bins, probability distribution of $\widetilde{w}_{i}(i=1-3), \widetilde{w}_{4}$, and $\widetilde{w}_{i}-\widetilde{w}_{j}$.
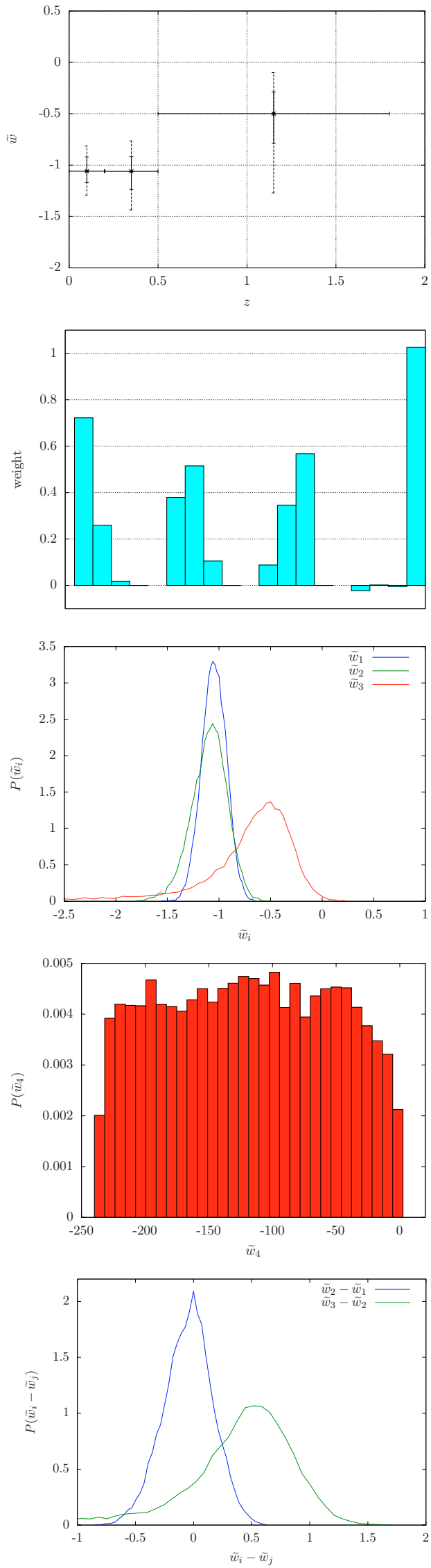

Fig. 4. Estimates of the uncorrelated dark energy EOS parameters using the strong prior. Same as Fig. 3 except using the strong prior. 

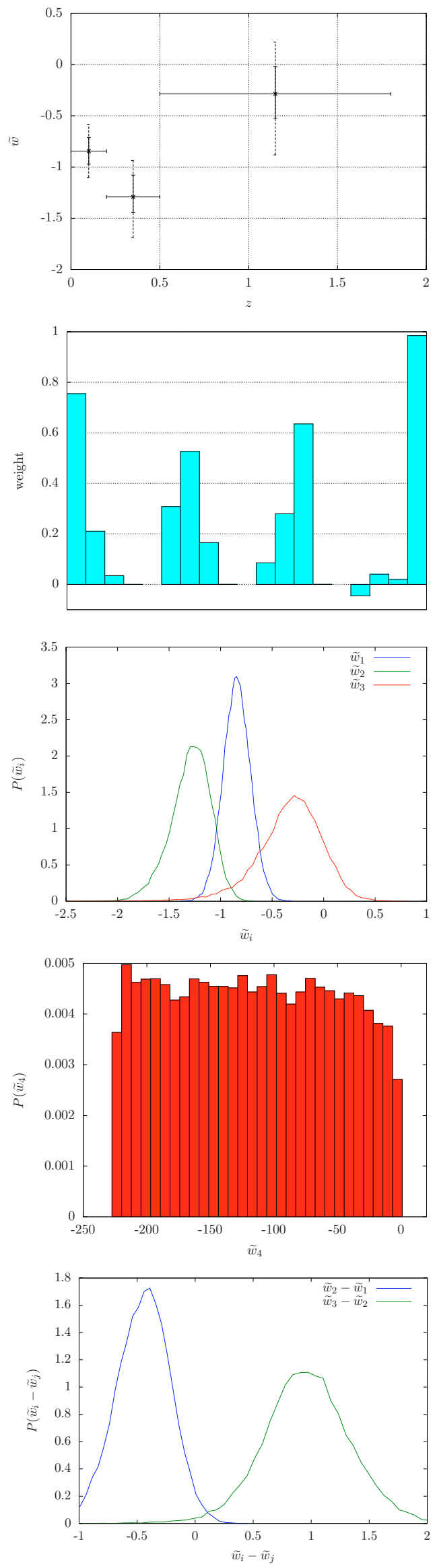

Fig. 5. Estimates of the uncorrelated dark energy EOS parameters using the strong prior. Same as Fig. 4 except BAO constraints were updated with the latest measurements (Percival et al. 2007). $w<-1$ (Percival et al. 2007). The BAO distance information lies in the second redshift bin, so including it leads to a smaller $\widetilde{w}_{2}$. And main data we used depends on the integration of the dark energy evolution, thus the decrease in $\widetilde{w}_{2}$ causes increases in $\widetilde{w}_{1}$ and $\widetilde{w}_{3}$.

The constraints on $\widetilde{w}_{4}$ are very weak. The uncertainty is so great that we plot its probability separately. This is due to three reasons. First, there are not enough samples of standard candles in the fourth bin, all of which are GRBs. From Table 1 it can be seen that the number ratio of third bin to the fourth bin is about 4. Second, as mentioned earlier, the estimate of the behavior of dark energy at high redshift depends on its behavior at low redshift; consequently, the uncertainty of EOS parameters at low redshift will be reflected on EOS parameters at high redshift. Therefore we get increasing errors as the redshift increases. Thirdly, the density ratio of dark energy to matter is given by (assuming a constant EOS parameter for dark energy)

$\frac{\rho_{x}}{\rho_{\mathrm{m}}}=\frac{\rho_{x 0}(1+z)^{3\left(1+w_{x}\right)}}{\rho_{\mathrm{m} 0}(1+z)^{3}} \approx 3(1+z)^{3 w_{x}}$.

For negative $w_{x}$, the ratio decreases as $z$ increases. For example, when $w_{x}=-1$, the ratio is about $1 / 9$ at $z=2$. At higher redshift, matter dominates over dark energy, then dark energy becomes less important in determining the cosmic expansion. Thus the constraints imposed on the behavior of dark energy by the expansion history become weak compared with that at low redshift where dark energy is important. Despite the large uncertainty in $\widetilde{w}_{4}$, there is indeed some restriction imposed by GRBs. From the probability plots of $\widetilde{w}_{4}$ in Figs. $3-5$, it can be seen that there is obviously a cut at about zero. In other words, it is most probable that the ratio in Eq. (26) continues to decrease at a redshift beyond 1.8. The probability cut at the left of -200 is due to the precision of the computer and can be viewed as the negative infinity. To get substantial constraints on the dark energy EOS beyond 1.8 , we need more GRB samples.

To see the overall improvement made by including GRB luminosity data, we calculate the figure of merit (FOM), which is defined by (Sullivan et al. 2007a,b)

$\mathrm{FOM}=\left[\sum_{i} \frac{1}{\sigma^{2}\left(\widetilde{w}_{i}\right)}\right]^{1 / 2}$.

For the the results presented in Fig. 5, FOM = 9.6. And if the GRB luminosity data are excluded, FOM $=8.8$.

\section{Summary}

We used a model-independent approach to constrain the evolution of dark energy. First, we separated the redshifts into 4 bins and assumed a constant EOS parameter for dark energy in each bin, then estimated the uncorrelated EOS parameters. We mainly used the SNe Ia and GRBs in our analysis. Other constraints from SDSS, 2dFGRS, HST, and WMAP are also included. Compared with the results obtained without including GRB luminosity data, the confidence interval of the third uncorrelated EOS parameter, whose contribution mostly comes from the third bin, is reduced significantly. Even though constraints at high redshift where we have only GRBs are very weak, from the obvious probability cut of the EOS parameter at about zero, we can infer that it is most probable that the ratio of dark energy to matter continues to decrease beyond redshift 1.8. To get substantial constraints at redshifts beyond SNe Ia more GRBs are needed. 
If the latest $\mathrm{BAO}$ measurements, which themselves favor a dark energy EOS of $w<-1$, are included, the results show some evidence for an evolving dark energy EOS. Otherwise, the results are consistent with the cosmological constant.

Acknowledgements. Shi Qi would like to thank Maurice HPM van Putten and Edna Cheung for helpful discussions and suggestions. This work was supported by the Scientific Research Foundation of the Graduate School of Nanjing University (for Shi Qi), the Jiangsu Project Innovation for Ph.D. candidates CX07B-039z (for Fa-Yin Wang), and the National Natural Science Foundation of China under Grant No. 10473023.

\section{References}

Abazajian, K., Adelman-McCarthy, J. K., Agüeros, M. A., et al. 2003, AJ, 126, 2081

Aldering, G., Althouse, W., Amanullah, R., et al. (SNAP Collaboration) 2004, PASP, submitted [arXiv: astro-ph/0405232]

Astier, P., Guy, J., Regnault, N., et al. 2006, A\&A, 447, 31

Allen, S. W., Schmidt, R. W., \& Fabian, A. C. 2002, MNRAS, 334, L11

Binetruy, P., Deffayet, C., Ellwanger, U., \& Langlois, D. 2000, Phys. Lett. B, 477,285

Capozziello, S., Cardone, V. F., Carloni, S., \& Troisi, A. 2003, Int. J. Mod. Phys. D, 12, 1969

Carroll, S. M., Duvvuri, V., Trodden, M., \& Turner, M. S. 2004, Phys. Rev. D, 70, 043528

Chevallier, M., \& Polarski, D. 2001, Int. J. Mod. Phys. D, 10, 213

Cooray, A. R., \& Huterer, D. 1999, ApJ, 513, L95

Copeland, E. J., Sami, M., \& Tsujikawa, S. 2006, Int. J. Mod. Phys. D, 15, 1753

Dai, Z. G., Liang, E. W., \& Xu, D. 2004, ApJ, 612, L101

Davis, T. M., Mörtsell, E., Sollerman, J., et al. 2007, ApJ, 666, 716

Deffayet, C. 2001, Phys. Lett. B, 502, 199

Di Girolamo, T., Catena, R., Vietri, M., \& Di Sciascio, G. 2005, JCAP, 04, 008

Dvali, G. R., Gabadadze, G., \& Porrati, M. 2000, Phys. Lett. B, 484, 112
Eisenstein, D. J., Zehavi, I., Hogg, D. W., et al. 2005, ApJ, 633, 560

Firmani, C., Ghisellini, G., Ghirlanda, G., \& Avila-Reese, V. 2005, MNRAS, 360, L1

Friedman, A. S., \& Bloom, J. S. 2005, ApJ, 627, 1

Ghirlanda, G., Ghisellini, G., Lazzati, D., \& Firmani, C. 2004, ApJ, 613, L13

Hamuy, M., Phillips, M. M., Suntzeff, N. B., Schommer, R. A., \& Maza, J. 1996, AJ, 112, 2408

Hawkins, E., Maddox, S., Cole, S., et al. 2003, MNRAS, 346, 78

Huterer, D., \& Cooray, A. 2005, Phys. Rev. D, 71, 023506

Huterer, D., \& Starkman, G. 2003, Phys. Rev. Lett., 90, 031301

Jha, S., Kirshner, R. P., Chaillis, P., et al. 2006, AJ, 131, 527

Lamb, D. Q., Ricker, G. R., Lazzati, D., et al. 2005, [arXiv: astro-ph/0507362]

Li, H., Su, M., Fan, Z., Dai, Z., \& Zhang, X. 2008, Phys. Lett. B, 658, 95

Liang, E.-W., \& Zhang, B. 2005, AJ, 633, 611

Linder, E. V. 2003, Phys. Rev. Lett., 90, 091301

Maartens, R. 2007, J. Phys. Conf. Ser., 68, 012046

Percival, W. J., Cole, S., Eisenstein, D. J., et al. 2007, MNRAS, 381, 1053

Perlmutter, S., Aldering, G., Goldhaber, G., et al. 1999, ApJ, 517, 565

Riess, A. G., Filippenko, A. V., Challis, P., et al. 1998, AJ, 116, 1009

Riess, A. G., Kirshner, R. P., Schmidt, B. P., et al. 1999, AJ, 117, 707

Riess, A. G., Strolger, L.-G., Casertano, S., et al. 2007, ApJ, 659, 98

Schaefer, B. E. 2007, ApJ, 660, 16

Spergel, D. N., Verde, L., Peiris, H. V., et al. 2003, ApJS, 148, 175

Spergel, D. N., Bean, R., Doré, O., et al. 2007, ApJS, 170, 377

Su, M., Fan, Z., \& Liu, B. 2006 [arXiv: astro-ph/0611155]

Sullivan, S., Cooray, A., \& Holz, D. E. 2007a, JCAP, 0709, 004

Sullivan, S., Sarkar, D., Joudaki, S., et al. 2007b [arXiv: 0709. 1150]

Tegmark, M., Blanton, M. A., Strauss. M. A., et al. 2004, ApJ, 606, 702

Wang, F. Y., \& Dai, Z.-G. 2006, MNRAS, 368, 371

Wang, F. Y., Dai, Z. G., \& Zhu, Z.-H. 2007, ApJ, 667, 1

Wang, Y., \& Mukherjee, P. 2007, Phys. Rev. D, 76, 103533

Wood-Vasey, W. M., Miknaitis, G., Stubbs, C. W., et al. 2007, ApJ, 666, 694

Wright, E. L. 2007, ApJ, 664, 633

Xu, D., Dai, Z., \& Liang, E. W. 2005, ApJ, 633, 603 